\title{
Interaction of Bradykinin with Substance P on Vascular Permeability and Pain Response
}

\author{
Manabu SHIBATA, Tsuyako OHKUBO, Hiroshi TAKAHASHI \\ and ${ }^{*}$ Reizo INOKI \\ Department of Pharmacology. Fukuoka Dental College. \\ 700 Ta, Sawara-ku, Fukuoka 814-01. Japan \\ *Department of Pharmacology, Osaka University Faculty of Dentistry, \\ 1-8, Yamadaoka, Suita, Osaka 565, Japan
}

Accepted May 8, 1986

\begin{abstract}
Combination of bradykinin with substance $P$ exerted synergistic effects on vascular permeability and pain response in mouse paw. Denervation of sciatic nerve reduced significantly bradykinin-induced vascular permeability, suggesting the involvement of sensory nerves. The bradykinin-induced vascular permeability was also reduced by intravenous injection of a substance $P$-antagonist. The results suggest that neuronal substance $P$ takes part in the action of bradykinin on inflammation and pain sensation.
\end{abstract}

Bradykinin has become of major interest recently as a chemical mediator of inflammation and pain generation. Previously, we have shown that bradykinin played an important role in formalin-induced pain response: substance $P$ was also involved in the formation of the pain response (1). It is reported that substance $P$ potentiated responses to bradykinin, serotonin, prostaglandin and ATP when tested on the dye-leakage response in rats (2). Bynke et al. demonstrated that injection of bradykinin into the vitreous chamber of the rabbit eye caused miosis and aqueous flare, and the response to bradykinin was reduced by substance $P$ antagonists (3). They suggest that bradykinin causes miosis and aqueous flare at least partly through local release of neuronal substance $P$. This paper presents the results indicating that a mutual relationship between bradykinin and substance $P$ may be of importance in inflammation and pain sensation.

Male ddY strain mice (20-25 g) were given an injection of bradykinin, substance $P$ or the mixture into a paw. To study the inflammatory reaction, a quantitative dyeleakage technique was used. Five min after injection of pontamine blue $(100 \mathrm{mg} / \mathrm{kg}$. i.v.), the mediator was administered into the paw. Subsequently, 5 min after, the inflamed hindpaw was cut out, chopped into smaller pieces and placed into $4 \mathrm{ml}$ of formamide. Twenty-four hr later, the amount of dye was measured according to the method of Gamse et al. (4). Pain threshold was determined by recording the durations (in sec) spent in licking or biting response against the inflamed forepaw during a 5 min period immediately after the mediator injection (5). In order to test whether substance $P$ interacts with the bradykinin action, mice were pretreated with substance $P$-antagonist $10 \mathrm{~min}$ before and the denervation of sciatic nerve was carried out $48 \mathrm{hr}$ before the experiment. The sciatic nerve of one side was cut in the midthigh under ether anesthesia. Statistical analysis was performed by Student's $t$-test.

Figure 1 shows the effect of bradykinin in combination with substance $P$ on pain response and vascular permeability. To study the effect of the combination, lower doses of bradykinin and substance $P$ were chosen since it produced only a small response. Bradykinin (5.23 nmol) and substance $P$ $(3.70 \mathrm{nmol})$ exerted synergistic effects on pain response. The effect of bradykinin (340 $\mathrm{pmol})$ with substance $\mathrm{P}(48 \mathrm{pmol})$ on vascular 

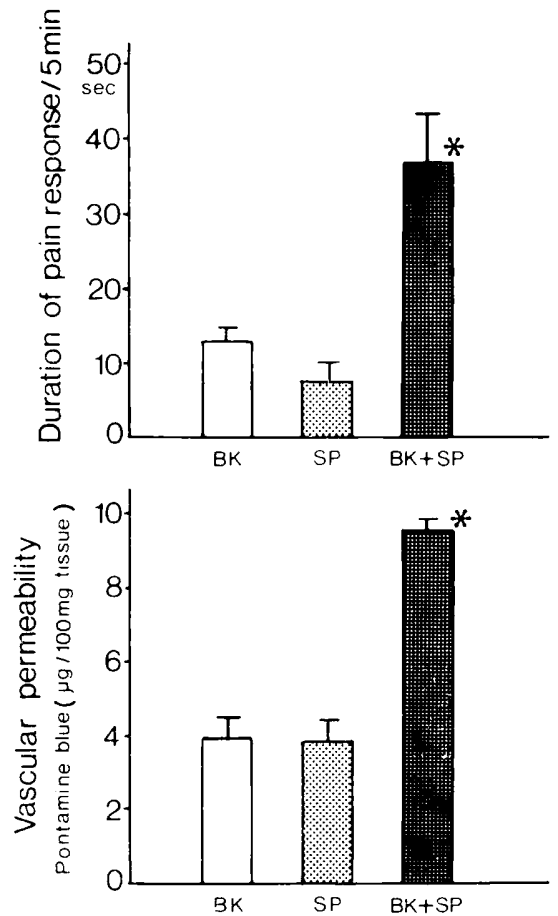

Fig. 1. Effect of bradykinin with substance $P$ on pain response and vascular permeability. BK bradykinin, SP: substance $\mathrm{P}, \mathrm{BK}+\mathrm{SP}$ : mixture. In studying the pain response, bradykinin (5.23 nmol) and substance $P(3.7 \mathrm{nmol})$ were used. In determining vascular permeability, bradykinin ( 340 pmol) and substance $\mathrm{P}$ (48 pmol) were used. Drugs were injected in a volume of $6.5, \mu l .{ }^{*}: P r 0.05$.

permeability was also greater than a simple summation of the two mediators. Denervation of the sciatic nerve and intravenous administration of (D-Arg $\left.{ }^{1}, \mathrm{D}-\mathrm{PrO}^{2}, \mathrm{D}-\mathrm{Trp}^{7,9}, \mathrm{Leu}^{11}\right)$ substance $P$ (substance $P$-antagonist) reduced significantly bradykinin-induced vascular permeability (Fig. 2).

From the facts that denervation of the sciatic nerve reduced dye-leakage induced by bradykinin (Fig. 2), it is suggested that bradykinin may act partly via functioning of sensory nerves. Furthermore, bradykinininduced dye-leakage was aiso reduced by substance P-antagonist. Gamse et al. reported that the substance $P$ content of the hind paw skin of rats was markedly decreased after cutting the saphenous nerve (4). These suggest that bradykinin acts by releasing a neurogenic mediator, possibly substance $P$.

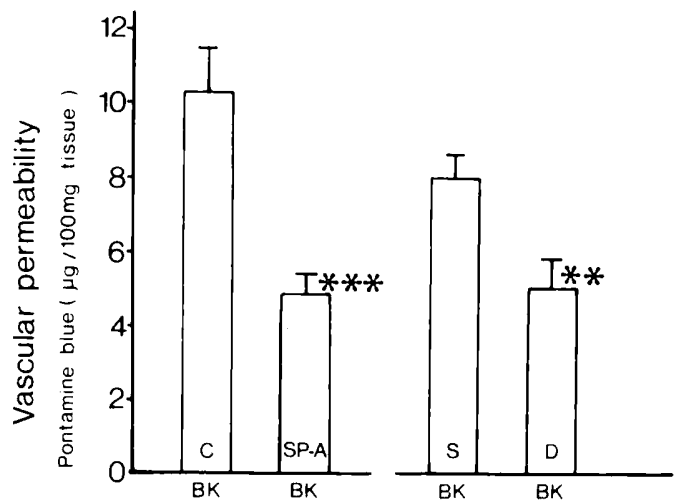

Fig. 2. Effects of denervation of the sciatic nerve and substance $P$-antagonist on bradykinin-induced vascular permeability. $C$ : saline control, SP-A: substance $P$-antagonist $(0.1 \mathrm{mg} / \mathrm{kg}$, i.v. $10 \mathrm{~min}$ before). S: sham operation, D: denervation of sciatic nerve (48 hr before) Bradykinin dose was 340 pmol. **: P. 0.01, ***: P 0.001 .

We and Jancsó et al. reported previously that there was a parallelism between the permeability and pain response in the process of neurogenic inflammation (1, 6). So it is reasonable that the synergistic effect of the drug combination on pain response was produced in the same manner as the effect on vascular permeability (Fig. 1). Therefore, it may be that there is a similar mechanism of regulation by bradykinin and substance $P$ on the process of pain sensation as well. Perhaps, noxious stimuli evoked the release of bradykinin and then bradykinin stimulated axonreflexive substance $P$ release, and these two mediators acted together. This hypothesis agrees with previous observations by Bynke et al. and Ueda et al. that substance $P$ is involved in the bradykinin-evoked response $(3,7)$. The results indicate that neuronal substance $P$ takes part in the action of bradykinin on inflammation and pain sensation.

\section{References}

1 Shibata, M.: Pharmacological study of formalininduced inflammatory pain response. Japan. J. Oral Biol. 27, 890--901 (1985)

2 Chahl, L.A.: Interactions of substance $P$ with putative mediators of inflammation and ATP. Eur. J. Pharmacol. 44, 45-49 (1977)

3 Bynke, G., Hakanson, R., Horig, J. and Leander, 
S.: Bradykinin contracts the pupilary sphincter and evokes ocular inflammation through release of neuronal substance P. Eur. J. Pharmacol. 91 , 469-475 (1983)

4 Gamse, P., Holzer, P. and Lembeck, F.: Decrease of substance $P$ in primary afferent neurones and impairment of neurogenic plasma extravasation by capsaicin. Br. J. Pharmacol. 68, 207-213 (1980)

5 Takahashi, H., Ohkubo, T., Shibata, M. and Naruse, S.: A modified formalin test for measuring analgesia in mice. Japan. J. Oral Biol. 26, 543-

\section{$548(1984)$}

6 Jancsó, N., Jancsó-Gábor, A. and Szolcsányi, J.: Direct evidence for neurogenic inflammation and its prevention by denervation and by pretreatment with capsaicin. Br. J. Pharmacol. 31, 138-151 (1967)

7 Ueda, N., Muramatsu, I. and Fujiwara, M.: Capsaicin and bradykinin-induced substance $P$ ergic responses in the iris sphincter muscle of the rabbit. J. Pharmacol. Exp. Ther. 230, 469-473 (1984) 